\title{
HMG-CoA reductase inhibitor lovastatin upregulates plasminogen activator production through RhoA-signaling in peritoneal cell line Met5A
}

\author{
Noriko Suzuki • Atsushi Imai
}

Received: 21 October 2009 /Accepted: 3 December 2009/Published online: 22 December 2009

(C) Springer-Verlag 2009

\begin{abstract}
This study was conducted to determine if hydroxymethylglutaryl-CoA (HMG-CoA) reductase inhibitor statin, known to protect postoperative adhesion in animal model, affect the expressing tissue-type plasminogen activator (tPA) in peritoneal cells in culture. Human peritoneal Met5A cells were used to examine the effects of hydrophobic statin lovastatin on the level of tPA. PA concentrations were measured by real-time polymerase chain reaction and enzyme-linked immunosorbent assay. Active RhoA form was also examined. Lovastatin caused concentrationdependent $\mathrm{PA}$ expression associated with fall of RhoA active level in Met5A cells. These lovastatin-induced changes were significantly overcome by the addition of geranylgeranyl pyrophosphate (intermediate of HMG-CoA pathway). A RhoA protein inhibitor $\mathrm{C} 3$ transferase mimicked the effects of lovastatin on the Met5A cells. These results suggest that lovastatin may be an effective stimulator of local peritoneal fibrinolytic activity, as it upregulates tPA expression in peritoneal Met5A cells through the reduction of RhoA geranylgeranylation. The extra-cholesterol lowering action of statin provides a new rationale to prevent peritoneal adhesion in postoperative patient.
\end{abstract}

Keywords Statin - Tissue-type plasminogen activator . RhoA $\cdot$ Peritoneal cells $\cdot$ Postoperative adhesion

\footnotetext{
N. Suzuki $\cdot$ A. Imai

Department of Obstetrics and Gynecology,

Gifu University School of Medicine,

Gifu, Japan

A. Imai $(\bowtie)$

Atsushi Imai at Institute of Endocrine-Related Cancer,

Matsunami General Hospital,

Kasamatsu,

Gifu 501-6062, Japan

e-mail: aimai@matsunami-hsp.or.jp
}

\section{Introduction}

Since the advent of surgery, peritoneal adhesions have been a significant and often inevitable postoperative complication $[1,2]$. It is a common consequence of serosal repair, occurring in $93-100 \%$ of patients following laparotomy, and may lead to serious complications such as intestinal obstruction, pelvic pain, and infertility. Peritoneal adhesions are defined as fibrous bands of tissue that join together organs that are normally separated. The plasma fibrinolytic system is primarily responsible for the degradation of fibrin. Tissue-type plasminogen activator (tPA) is considered to play an important role in the onset of the extrinsic fibrinolytic route [3, 4]. It has been proposed that the persistence of fibrin, due to impaired tPA activity, results in the formation of adhesions between damaged serosal surfaces.

Hydroxymethylglutaryl-CoA (HMG-CoA) reductase is the rate-limiting enzyme in the conversion of HMG-CoA to mevalonate, an intermediate in the de novo synthesis of cholesterol [5-7]. Apart from the conversion of mevalonate to cholesterol via a number of enzymatic steps, several lipid isoprenoid intermediate such as geranylgeranyl pyrophosphate (GGPP) and farnesyl pyrophosphate are enzymatically generated from mevalonate. Small guanosine triphosphate (GTP)-binding proteins (G-protein), including Rho, that play pivotal roles in normal and oncogenic signaling, undergo posttranslational modification, termed isoprenylation, allow the attachment of $\mathrm{G}$ proteins to internal cell membranes by means of a lipid anchor [5]. HMG-CoA reductase inhibitors, commonly referred to as statins including lovastatin, are in wide use for the treatment of hypercholesterolemia.

Recent studies have elucidated additional effects of statin beyond their impact on serum cholesterol levels [5-9]. 
Aarons et al. reports that statins decrease postoperative adhesion by increasing peritoneal fibrinolytic activity in rat model [3]. This beneficial effect beyond lowering cholesterol might be due in part to the ability of statins to inhibit the synthesis of GGPP that interferes with G-protein-mediated RhoA activation $[10,11]$ using peritoneal cells derived from the omentum. In this study, we investigated whether lovastatin increases fibrinolytic potential by the induction of tPA through inhibiting the RhoA-dependent pathway in human peritoneal Met5A cells.

\section{Materials and methods}

\section{Materials}

Lovastatin and GGPP were obtained from Calbiochem (Darmstat, Germany) and Sigma (St. Louis, MO, USA), respectively. C3 transferase was a product of Cytoskelton (Denver, CO, USA). Human tPA enzyme-linked immunosorbent assay (ELISA) kit was purchased from AssayPro (St. Charles, MO, USA). All other chemicals were of reagent grade. This study did not require approval of our institutional review board because it did not imply active involvement of patients.

\section{Cell culture}

Human mesothelial (peritoneal) Met5A cells were obtained from ATCC (Manassas, VA, USA). The Met5A cells were cultured in a $5 \% \mathrm{CO}_{2}$ humidified atmosphere in Medium199 with 10\% fetal bovine serum, $75 \mathrm{mM}$ L-glutamine, $1.25 \mathrm{~g} / 1$ sodium bicarbonate, $3.3 \mathrm{nM}$ epidermal growth factor, $400 \mathrm{nM}$ hydrocortisone, $870 \mathrm{nM}$ insulin, $20 \mathrm{mM}$ HEPES, 100 IU penicillin, and streptomycin. Lovastatin was converted from its inactive form to active form following the manufacturer's instructions by dissolving $52 \mathrm{mg}$ of the compound in $1.04 \mathrm{ml}$ of ethanol and then adding $813 \mu \mathrm{l}$ of $1 \mathrm{~N} \mathrm{NaOH}$. The resulting solution was neutralized with $1 \mathrm{~N} \mathrm{HCl}$ to a $\mathrm{pH}$ of 7.2 and brought up to a volume of $13 \mathrm{ml}$ with distilled water. The stock solution $(10 \mathrm{mM})$ was stored at $-20^{\circ} \mathrm{C}$ [12]. At least three separate cell culture experiments were performed in duplicate (using two wells), and each experiment gave similar results.

tPA protein

Met5A cells $\left(1 \times 10^{5}\right)$ were plated into 12 -well plate and treated with the appropriate concentration of the chemicals to be tested for $18 \mathrm{~h}$ (overnight interval) where cells were in sub-confluent. Cells were lysed in phosphate buffer solution by freeze-thawing followed by brief sonication. The tPA concentration in cell lysate was determined with
AssayMax tPA ELISA kit. The absorbance (optical density) was measured at $450 \mathrm{~nm}$.

tPA mRNA

Total RNA was isolated with CellAmp ${ }^{\mathrm{TM}}$ Direct RNA Prep kit for One Step reverse transcription (RT)-polymerase chain reaction (PCR; Takara Bio.Co., Ohtsu, Japan) according to the manufacturer's protocol. The designed oligonucleotide sequences were verified to amplify unique sequence, tPA: (F) 5'-AGCAGGCCCTGTACTTCTC and (R) 5'-TCTGCAGTAGTTGTGGTTCC, ß-actin (as a housekeeping gene): (F) 5'-AGAAAATCTGGCACCA CACC and (R) 5'-AGAGGCGTACAGGGATAGCA, respectively. Real-time PCR analysis was performed using a One Step SYBR PrimeScript RT-PCR Kit (Takara Bio. Co.) and analyzed with a Takara Smart cycler system using a twostep program consisting of $5 \mathrm{~s}$ at $95^{\circ} \mathrm{C}$ and $30 \mathrm{~s}$ at $60^{\circ} \mathrm{C}$ for 40 cycles. $\beta$-Actin was included as an endogenous normalization control.

\section{Measurement of active Rho}

The activation state of Rho-GTP was measured using G-LISA RhoA Activation Assay Biochem Kit from Cytoskelton. The absorbance was measured at $490 \mathrm{~nm}$.

\section{Statistics}

The variables followed normal distribution, and statistical analysis was performed with the Student's $t$ test, and a $P$ value of less than 0.05 was considered significant.

\section{Results}

Lovastatin showed the effects of dose-dependent increase in the production of tPA (Fig. 1). To determine whether the lovastatin effects were mediated by the isoprenylation, we incubated Met5A cells with GGPP, a downstream intermediate of mevalonate in the presence of lovastatin and measured tPA production. The effect of lovastatin to increase tPA production was overcome by addition of GGPP, indicating that lovastatin-induced inhibition of GGPP synthesis is critical for the increase of tPA (Fig. 2). Lovastatin might increase tPA production through intervention in the GGPP pathway.

Rho is known to be one of the most important isoprenylated proteins. To determine whether lovastatin effects on tPA result from the inhibition of Rho proteins, we incubated Met5A cells with C3 exoenzyme, a specific inhibitor for RhoGTPase. As shown in Fig. 2, C3 completely mimicked the effect of lovastatin on tPA 


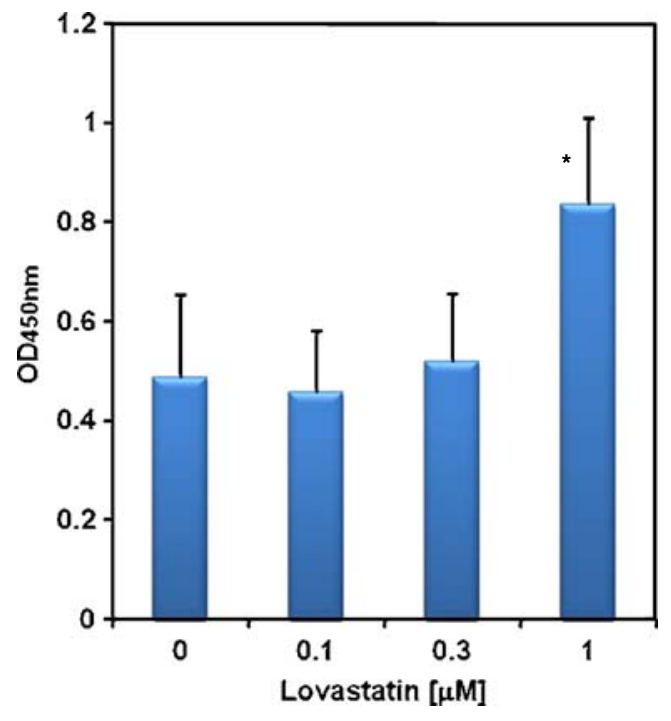

Fig. 1 Dose-dependency of lovastatin-stimulated tissue-type plasminogen activator (tPA) production. Met5A cells were treated with 0 (vehicle), 0.1, 0.3, or $1 \mu \mathrm{M}$ lovastatin for $18 \mathrm{~h}$. tPA protein content of cell lysate was measured by tPA enzyme-linked immunosorbent assay kit. Values are expressed as means optical density $(450 \mathrm{~nm}) \pm \mathrm{SD}$ of three independent experiments performed in duplicate determination. $* P<0.05$ versus control

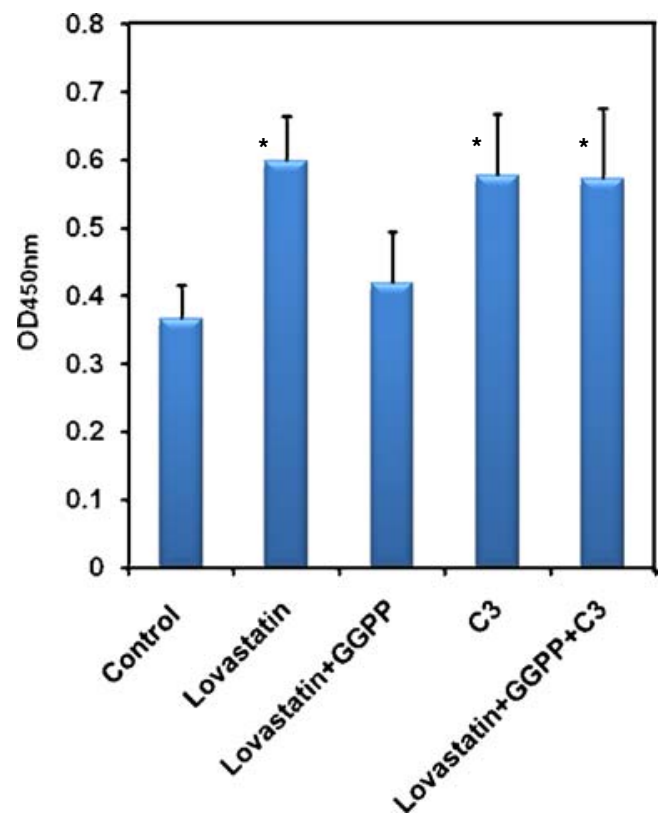

Fig. 2 Tissue-type plasminogen activator (tPA) protein level in Met5A cells exposed to geranylgeranyl pyrophosphate (GGPP) or C3 transferase. Met5A cells were exposed to lovastatin $(1 \mu \mathrm{M})$, lovastatin and GGPP $(1 \mu \mathrm{M}), \mathrm{C} 3(1 \mu \mathrm{g} / \mathrm{ml})$, or lovastatin, GGPP, and $\mathrm{C} 3$ for $18 \mathrm{~h}$. tPA protein content of cell lysate was measured by tPA enzyme-linked immunosorbent assay kit. Values are expressed as means optical density $(450 \mathrm{~nm}) \pm \mathrm{SD}$ of three independent experiments performed in duplicate determination. $* P<0.05$ versus control production and also blocked the reversion of lovastatin effects by GGPP. These results suggest that the effect of the lovastatin on TPA production was through the reduction of RhoA geranylgeranylation.

To demonstrate that the effects of lovastatin to increase the level of medium IPA were not due to stimulation of their cellular secretion, tPA mRNA level was examined. The RTPCR analysis revealed higher levels of tPA mRNA level in lovastatin-treated Met5A cells (Fig. 3). GGPP totally abolished the effect of lovastatin on IPA mRNA expression. The addition of exoenzyme $\mathrm{C} 3$ transferase reversed tPA mRNA level to control from those observed in lovastatinor GGPP-treated cells. These results were good agreeable with protein level changes. Lovastatin had no effects on the levels of plasminogen activator inhibitor (PAI) protein (data not shown).

Rho protein's cycle between an active (GTP-bound) form and an inactive (guanosine diphosphate (GDP)-bound) form is important for Rho's interaction with upstream regulators and downstream effectors. In Fig. 2, we showed C3 reversed the effect of lovastatin on tPA production. To determine whether lovastatin decreases the RhoA active form, we measured directly RhoA active form by using ELISA. As shown in Fig. 4, lovastatin decreased the active form of RhoA. This lovastatin-induced change was attenuated by the addition of GGPP. A RhoA protein inhibitor C3 transferase mimicked the effects of lovastatin to reduce RhoA active form in the Met5A cells. These results indicate that geranylgeranylation of Rho to be active GTP-form is required for reversion of the effect of lovastatin by GGPP.

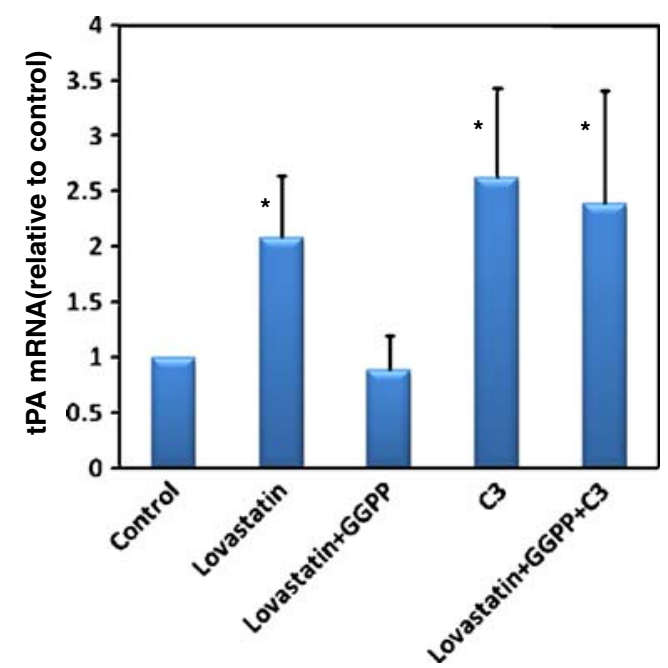

Fig. 3 Tissue-type plasminogen activator mRNA level in Met5A cells exposed to lovastatin, geranylgeranyl pyrophosphate (GGPP), or C3. Total RNA was extracted for real-time polymerase chain reaction analysis from the cells exposed to lovastatin $(1 \mu \mathrm{M})$, lovastatin and GGPP $(1 \mu \mathrm{M}), \mathrm{C} 3(1 \mu \mathrm{g} / \mathrm{ml})$, or lovastatin, GGPP, and C3 for $18 \mathrm{~h}$. As relative to control $\pm \mathrm{SD}$ of three independent experiments performed in duplicate determination. ${ }^{*} P<0.05$ versus control 


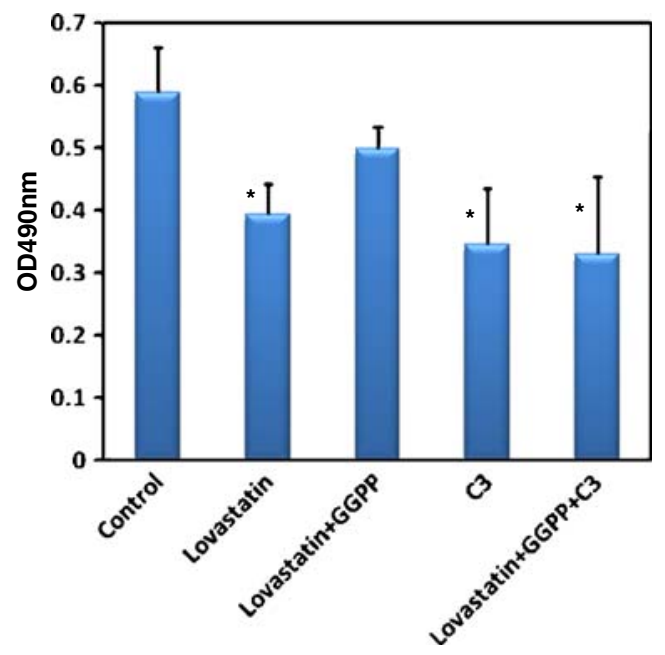

Fig. 4 RhoA activity in Met5A cells exposed to lovastatin. Met5A cells were exposed to lovastatin $(1 \mu \mathrm{M})$, lovastatin and geranylgeranyl pyrophosphate (GGPP; $1 \mu \mathrm{M}), \mathrm{C} 3(1 \mu \mathrm{g} / \mathrm{ml})$, or lovastatin, GGPP, and $\mathrm{C} 3$ for $18 \mathrm{~h}$. Values are expressed as means optical density $(490 \mathrm{~nm}) \pm \mathrm{SD}$ of three independent experiments performed in duplicate determination. $* P<0.05$ versus control

\section{Discussion}

Statins are synthetic HMG-CoA reductase inhibitors that are potent suppressors of cholesterol biosynthesis in humans. Recently, increasing evidence suggests that statins exert pleiotropic effects independent of cholesterol reduction, in particular, in animal models [3, 7]. For postoperative adhesion prevention, several in vitro studies indicated that statins increased fibrinolytic activity in human peritoneal cells independent of cholesterol lowering $[3,7]$. In this study, we showed that lovastatin caused a dose-dependent increase in tPA production within a range of concentrations $0.1-1 \mu \mathrm{M}$ using Met5A cells; lovastatin had no effect on PAI levels. More than $5 \mu \mathrm{M}$, lovastatin had possibility of a cytotoxic effect of lovastatin in the Met-5A cells, as previously reported [8]. By inhibition of HMG-CoA reductase, statins block the reduction of HMG-CoA to mevalonate which is the precursor molecule for the generation of the isoprenoid intermediates farnesylpyrophosphate and GGPP. The effect of lovastatin to induce tPA production could be suppressed by GGPP.

The use of this cell line differs in many ways from primary cultured mesothelial cells, for example, from omental tissues or cells derived from peritoneal fluid. They differ in the release of fibrinolytic factors upon stimulation. However, consideration of poor knowledge regarding mechanism and treatment against postoperative peritoneal adhesion led us to study the Met5A cells for fibrinolytic PA activity because of no information using Met5A cells.

The Rho family is one of the most important geranylgeranylated proteins. The Rho belongs to the Ras superfamily of GTPases that cycle between an inactive cytosolic GDP- bound form and an active membrane GTP-bound form. GTP-bound Rho recognizes and interacts with its cofactor to initiate a downstream response [13]. Our study demonstrated that the effect of lovastatin on TPA was reproduced by the C3 exoenzyme, a specific inhibitor of the Rho proteins. We also observed that lovastatin reduced the level of active RhoA. Furthermore, $\mathrm{C} 3$ blocked the reversion of lovastatin effects induced by GGPP, thus suggesting the involvement of the RhoA activation in the signaling pathway of lovastatin on tPA.

These results indicated that by preventing membrane interaction, statins rapidly inactivate RhoA, leading to increased tPA expression (protein and mRNA levels) and activity in peritoneal mesothelial cells. The mechanism linking Rho proteins to fibrinolysis is not fully elucidated. An involvement of the cytoskeleton could be assumed because Rho proteins are known to regulate the organization of the cytoskeleton and the formation. In the course of examination of statin's effects on balance of tPA-PA inhibitor (PAI), we found actin skeleton perturbation and morphology alterations of peritoneal cells in response to statin (data not shown). Statin acts on peritoneal cells by the possible mechanism involving RhoA-modified actin filament reorganization $[3,14,15]$.

In summary, our study describes that lovastatin increased tPA production by a mechanism involving geranylgeranylmodified intermediates in Met5A cells in culture. The proposed mechanism is summarized in Fig. 5. RhoA would play a central role in signal transduction for peritoneal cells

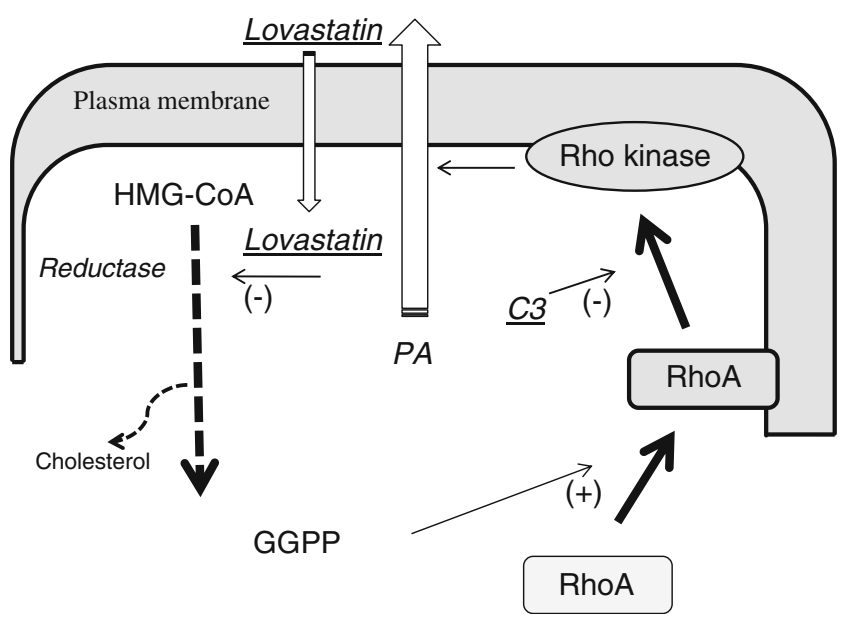

Fig. 5 The proposed effects of lovastatin on plasminogen activator (PA) upregulation. Hydroxymethylglutaryl (HMG)-CoA reductase stimulates the synthesis of mevalonate and isoprenoid intermediate geranylgeranyl pyrophosphate (GGPP). GGPP catalyzes the geranylgeranylation of RhoA, a key step in the translocation of RhoA from cytoplasm to the plasma membrane. The active membrane-bound RhoA initiates downstream signaling via Rho kinase to downregulate PA. HMG-CoA inhibitor lovastatin reduces RhoA activation and fibrinolytic activity by preventing the membrane localization and activation of PA production 
and be therefore a powerful target in molecular therapy for non-cholesterol lowering therapy of statins. Statins may be an alternative effective stimulator of local peritoneal fibrinolytic activity, as it increases tPA expression in peritoneal Met5A cells by a mechanism involving GGPP-mediated signaling. As prevention of postoperative adhesions is still a problem that has yet not been solved, the results of this in vitro experiment may give new insight into peritoneal adhesion prevention and adhesion development in postoperative patient, although whether statins prove to be therapeutically efficacious in adhesion prevention in human remains to be investigated. A more profound understanding of the molecular and cellular components involved in the formation of adhesions will propel a safe and efficient strategy of adhesion prevention using statins.

Conflict of interest No author has any conflict of interest.

\section{References}

1. DiZeregal G, Campeau J (2001) Peritoneal repair and postsurgical adhesion formation. Hum Reprod Update 7:547-555

2. González-Quintero V, Cruz-Pachano F (2009) Preventing adhesions in obstetric and gynecologic surgical procedures. Rev Obstet Gynecol 2:38-45

3. Aarons C, Cohen P, Gower A, Reed K, Leeman S, Stucchi A, Becker J (2007) Statins (HMG-CoA reductase inhibitors) decrease postoperative adhesions by increasing peritoneal fibrinolytic activity. Ann Surg 245:176-184
4. Sulaiman H, Dawson L, Laurent G, Bellingan G, Herrick S (2002) Role of plasminogen activators in peritoneal adhesion formation. Biochem Soc Transact 30:126-131

5. Endres M, Laufs U (2004) Effects of statins on endothelium and signaling mechanisms. Stroke 35:2708-2711

6. Fritz G (2005) HMG-CoA reductase inhibitors (statins) as anticancer drugs (review). Int J Oncol 27:1401-1409

7. Liao J, Laufs U (2005) Pleiotropic effects of statins. Annu Rev Pharmacol Toxicol 45:89-118

8. Agarwal B, Halmos B, Feoktistov A, Protiva P, Ramey W, Chen M, Pothoulakis C, Lamont J, Holt P (2002) Mechanism of lovastatininduced apoptosis in intestinal epithelial cells. Carcinogenesis 23:521-528

9. Fuchs D, Berges C, Opelz G, Daniel V, Naujokat C (2008) HMGCoA reductase inhibitor simvastatin overcomes bortezomibinduced apoptosis resistance by disrupting a geranylgeranyl pyrophosphate-dependent survival pathway. Biochem Biophys Res Commun 374:309-314

10. Haslinger B, Goedde M, Toet K, Kooistra T (2002) Simvastatin increases fibrinolytic activity in human peritoneal mesothelial cells independent of cholesterol lowering. Kidney Int 62:16111619

11. Haslinger B, Kleemann R, Toet K, Kooistra T (2003) Simvastatin suppresses tissue factor expression and increases fibrinolytic activity in tumor necrosis factor-alpha-activated human peritoneal mesothelial cells. Kidney Int 63:2065-2074

12. Keyomarsi K, Sandoval L, Band V, Pardee A (1991) Synchronization of tumor and normal cells from $\mathrm{G}_{1}$ to multiple cell cycles by lovastatin. Cancer Res 51:3602-3609

13. Narumiya S, Tanji M, Ishizaki T (2009) Rho signaling, rock and mdia1, in transformation, metastasis and invasion. Cancer Metastasis Rev 28:65-76

14. Farina H, Bublik D, Alonso D, Gomez D (2002) Lovastatin alters cytoskeleton organization and inhibits experimental metastasis of mammary carcinoma cells. Clin Exp Metastasis 19:51-559

15. Van der Wal J, Jeekel J (2007) The use of statins in postoperative adhesion prevention. Ann Surg 245:185-186 